\title{
Is GBT $1355+5439$ a dark galaxy?
}

\author{
T. A. Oosterloo ${ }^{1,2}$, G. H. Heald ${ }^{1}$, and W. J. G. de Blok ${ }^{1,3}$ \\ 1 Netherlands Institute for Radio Astronomy (ASTRON), Postbus 2, 7990 AA Dwingeloo, The Netherlands \\ e-mail: oosterloo@astron.nl \\ 2 Kapteyn Astronomical Institute, University of Groningen, Postbus 800, 9700 AA Groningen, The Netherlands \\ 3 Astrophysics, Cosmology and Gravity Centre (ACGC), Department of Astronomy, University of Cape Town, Private Bag X3, \\ 7701 Rondebosch, South Africa
}

Received 26 May 2013 / Accepted 14 June 2013

\section{ABSTRACT}

\begin{abstract}
We present H I imaging of GBT $1355+5439$ performed with the Westerbork Synthesis Radio Telescope. This is a dark H I object recently discovered close to the nearby galaxy M101. We find GBT $1355+5439$ to be an H I cloud $5 \times 3$ arcmin in size. The total $\mathrm{H}$ I image and the kinematics show that the cloud consists of condensations that have small $\left(\sim 10 \mathrm{~km} \mathrm{~s}^{-1}\right)$ motions with respect to each other. The column densities of the H I are low; the observed peak value is $7.1 \times 10^{19} \mathrm{~cm}^{-2}$. The velocity field shows a mild velocity gradient over the body of GBT $1355+5439$, possibly due to rotation, but it may also indicate large-scale radial motions. Although our data are limited in sensitivity, at all positions the H I velocity dispersion is higher than $5 \mathrm{~km} \mathrm{~s}^{-1}$ and no narrow, cold, H I component is seen. Because its distance is not known, we considered various possibilities for the nature of GBT 1355+5439. Both the scenarios that it is a tidal remnant near M101 and that it is a dark dwarf companion of M101 meet difficulties. Neither do the data fit the properties of known compact high-velocity clouds in the Galactic halo exactly, but we cannot entirely exclude this option and deeper observations are required. We also considered the possibility that GBT $1355+5439$ is a gas-rich dark minihalo in the outer regions of the Local Group. Interestingly, it would then have similar properties as the clouds of a proposed Local Group population recently found in the ALFALFA survey. In this case, the H I mass of GBT $1355+5439$ would be about a few times $10^{5} M_{\odot}$, its size about $1 \mathrm{kpc}$, and the dynamical mass $M_{\mathrm{dyn}}>5 \times 10^{7} M_{\odot}$. However, if GBT 1355+5439 is a dark Local Group object, the internal kinematics of the $\mathrm{H}$ I appears to be different from that of gas-dominated, almost dark galaxies of similar size.
\end{abstract}

Key words. galaxies: dwarf - radio lines: galaxies

\section{Introduction}

One of the puzzles of extragalactic astronomy is the formation of the smallest galaxies. Because of their shallow dark matter potentials, they are very delicate and sensitive systems, and modelling their evolution requires an accurate description and balance of all physics. A number of years ago, there appeared to be a large discrepancy between predictions and observations regarding the number of dwarf galaxies (Klypin et al. 1999; Moore et al. 1999). However, due to a large amount of theoretical work and new optical surveys (see McConnachie 2012), the gap between observations and theory has considerably decreased.

Nevertheless, some important questions remain. A key feature of galaxy formation is that star formation becomes increasingly inefficient for progressively smaller galaxies. This leads to the question whether there is a minimum halo mass below which no stars form, and, if there were such a limit, whether there are starless objects, but that are nevertheless visible in other tracers, e.g. through their cold gas (e.g. Sternberg et al. 2002; Maloney \& Putman 2003; Bovill \& Ricotti 2011). Several searches for such gas-rich "dark galaxies" have been made. Although a number of objects have been found that are almost dark, there is no observational evidence for an extensive population of gas-rich dark objects, either in the Local Group or in other nearby systems (Zwaan \& Briggs 2000; Meyer et al. 2004; Pisano et al. 2007; Chynoweth et al. 2011, but see Giovanelli et al. 2010, for an interesting new possibility).

Recently, a deep survey of a region around the nearby galaxy M101, conducted in single-dish H I (GBT) and in the optical, revealed an, at the GBT resolution of 9 arcmin, unresolved isolated H I cloud (GBT 1355+5439, hereafter GBT 1355) near M101. It has no optical counterpart, even in very deep optical images (Mihos et al. 2012, 2013). The immediate question is, of course, whether the object is a dark galaxy. Answering this question requires spatially resolved $\mathrm{H}$ I data, and here we report on interferometric observations that have allowed us to image the distribution and kinematics of the object. Although the detection of the HI in GBT 1355 is fairly faint, a number of interesting features are detected that may give some clues to the nature of this object.

\section{Data}

The neutral hydrogen in GBT 1355 was imaged using a standard 12-h observation with the WSRT on January 29, 2013. A $10 \mathrm{MHz}$ bandwidth was used, divided into 2048 channels, giving a nominal velocity resolution of about $1 \mathrm{~km} \mathrm{~s}^{-1}$. Standard WSRT calibration and data reduction procedures were applied using the MIRIAD package (Sault Teuben \& Wright 1995).

The initial inspection of the data immediately made clear that the H I column densities in GBT 1355 are below $10^{20} \mathrm{~cm}^{-2}$ : in data cubes made with standard full spatial resolution only a hint of $\mathrm{HI}$ is visible. Only when the data are tapered to lower spatial and velocity resolution, a clear detection of GBT 1355 is made. A good compromise between resolution and signal-to-noise ratio $(\mathrm{S} / \mathrm{N})$ was found by smoothing the data spatially to a resolution of 55 arcsec. 


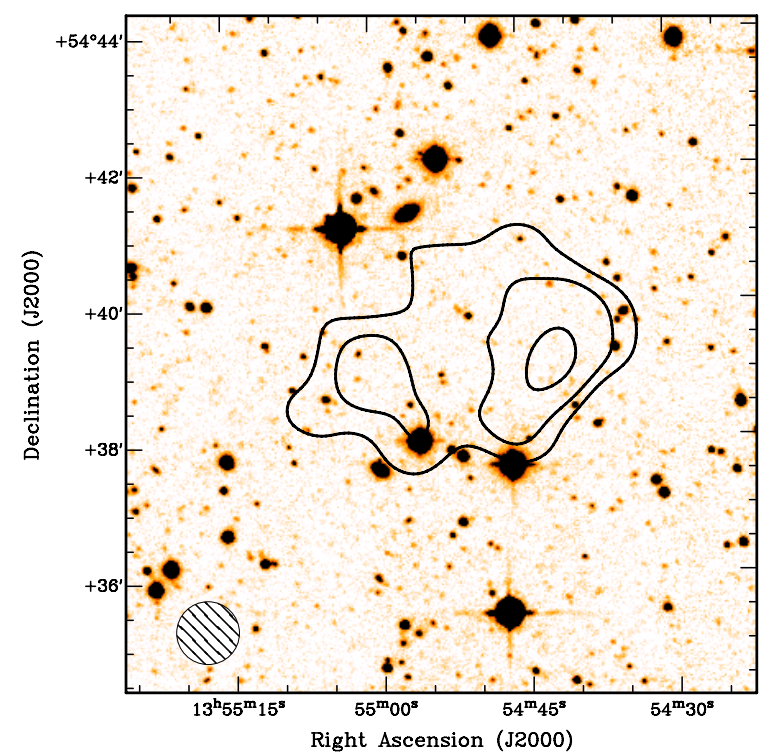

Fig. 1. Integrated $\mathrm{H}$ I contours on the deep $V$-band optical image from Mihos et al. (2013), kindly provided to us by Chris Mihos. The 1- $\sigma$ limiting surface brightness in this optical image is $\mu_{V}=29 \mathrm{mag} \mathrm{arcsec}^{-2}$. H I contour levels are $1.5,3$ and $6 \times 10^{19} \mathrm{~cm}^{-2}$.

To construct the total $\mathrm{HI}$ image, the data were binned to a velocity resolution of $16.4 \mathrm{~km} \mathrm{~s}^{-1}$. This was done so that the $\mathrm{HI}$ is found in only $1-2$ channels, maximising the $\mathrm{S} / \mathrm{N}$ for detection. The noise in this data cube is $0.5 \mathrm{mJy}_{\text {beam }}^{-1}$ and the $5-\sigma$ detection limit per 3D resolution element is $1.45 \times 10^{19} \mathrm{~cm}^{-2}$. The total H I image obtained in this way is shown in Fig. 1.

The overall shape of GBT 1355 is roughly elliptical, oriented east-west, extending $5 \times 3$ arcmin. The H I distribution shows two concentrations with fainter $\mathrm{HI}$ in between. The observed total H I flux is $1.05 \pm 0.15 \mathrm{Jy} \mathrm{km} \mathrm{s}^{-1}$. This is basically the same as derived by Mihos et al. (2012) from the GBT data, that have a spatial resolution of 9!1 (roughly the size of Fig. 1). This indicates that all the HI detected with the GBT is represented in Fig. 1.

Figure 1 confirms that the HI column densities are indeed low: the peak value is $7.1 \times 10^{19} \mathrm{~cm}^{-2}$ at the western side of the object; on the eastern side a second peak is observed with a column density of $4.9 \times 10^{19} \mathrm{~cm}^{-2}$. The range of column densities observed is lower than the critical column density below which no star formation is expected to occur (e.g., Schaye 2004).

The kinematics of the H I was studied by fitting Gaussians to the spectra of a data cube made with the same spatial tapering as used above, but now with a velocity resolution of $4.2 \mathrm{~km} \mathrm{~s}^{-1}$. The velocity field is shown in Fig. 2. This figure shows that there is a $\mathrm{N}-\mathrm{S}$ velocity gradient of about $15 \mathrm{~km} \mathrm{~s}^{-1}$ over the object, more or less perpendicular to the morphological major axis. This velocity gradient could indicate overall rotation of the $\mathrm{H} \mathrm{I}$, but given that the gradient is aligned with the minor axis of the H I body, it may as well indicate a radial flow (in or out) of the gas. Below we show that at some locations the spectra show multiple components. This means that the velocity field only crudely represents the overall kinematics. The heliocentric systemic velocity was found to be $V_{\text {sys }}=208 \pm 5 \mathrm{~km} \mathrm{~s}^{-1}$, consistent with the value of $210 \mathrm{~km} \mathrm{~s}^{-1}$ found by Mihos et al. (2012).

Figure 3 gives the velocity dispersion map. In the north the lowest dispersions are found, with values between 5 and $8 \mathrm{~km} \mathrm{~s}^{-1}$, while in the south dispersions have values above $20 \mathrm{~km} \mathrm{~s}^{-1}$. In the western part the dispersions are intermediate with values around $15 \mathrm{~km} \mathrm{~s}^{-1}$. Nowhere dispersions below

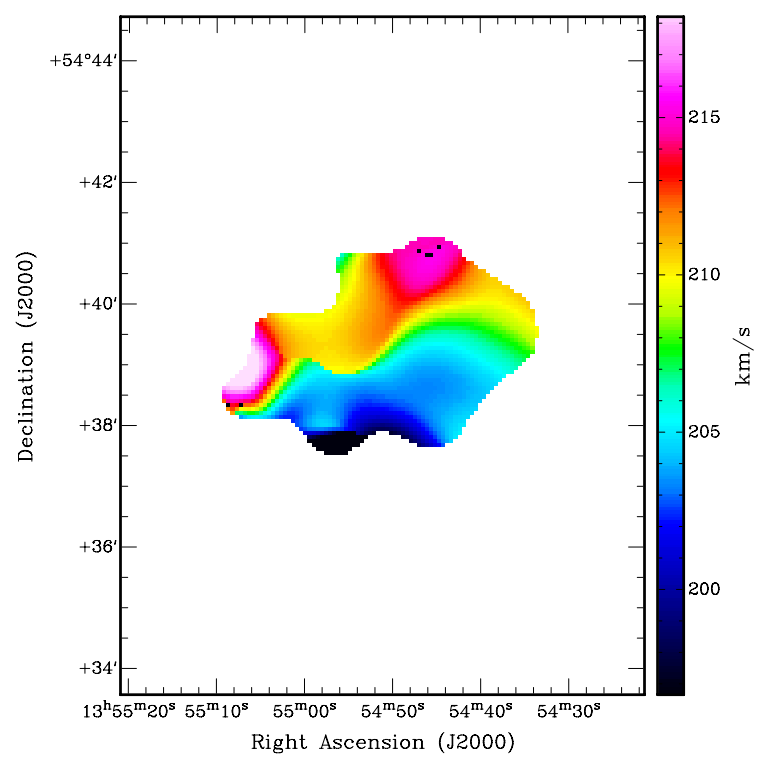

Fig. 2. Velocity (heliocentric) field of the H I in GBT 1355.

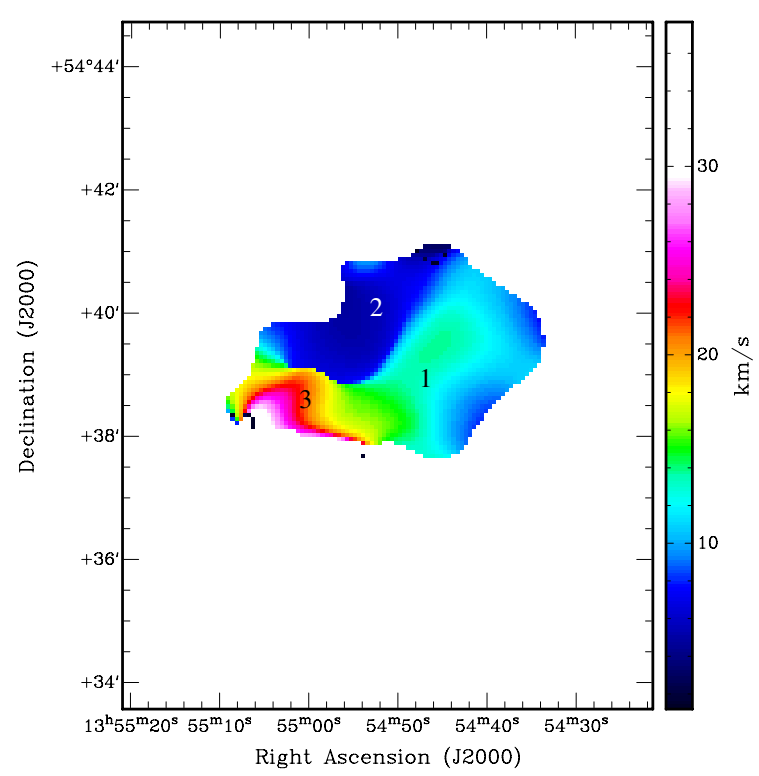

Fig. 3. Velocity dispersion of the HI in GBT 1355. The numbers indicate where the spectra given in Fig. 4 were taken.

$5 \mathrm{~km} \mathrm{~s}^{-1}$ are detected, even though the spectral resolution would allow detecting dispersions as low at $1.5 \mathrm{~km} \mathrm{~s}^{-1}$.

There is quite some structure in the spatial distribution of the dispersions, and there appears to be a fairly sharp transition between the relatively narrow profiles in the north and the wide profiles in the south. There are indications that the high dispersions in the south are due to fitting a single Gaussian to profiles that are a sum of two narrower components. This is illustrated in Fig. 4 where three spectra are shown. Spectrum 1 is taken in the western peak of the $\mathrm{HI}$ distribution where the intermediate dispersions are found, while spectrum 2 is taken in the region of lower velocity dispersion. Spectrum 3 is taken from the region with highest dispersions and appears to be composed of two narrower components. The most likely explanation is that GBT 1355 contains a small number of gas concentrations that have small random motions with respect to each other and that in the southern part we see two such concentrations superposed. 


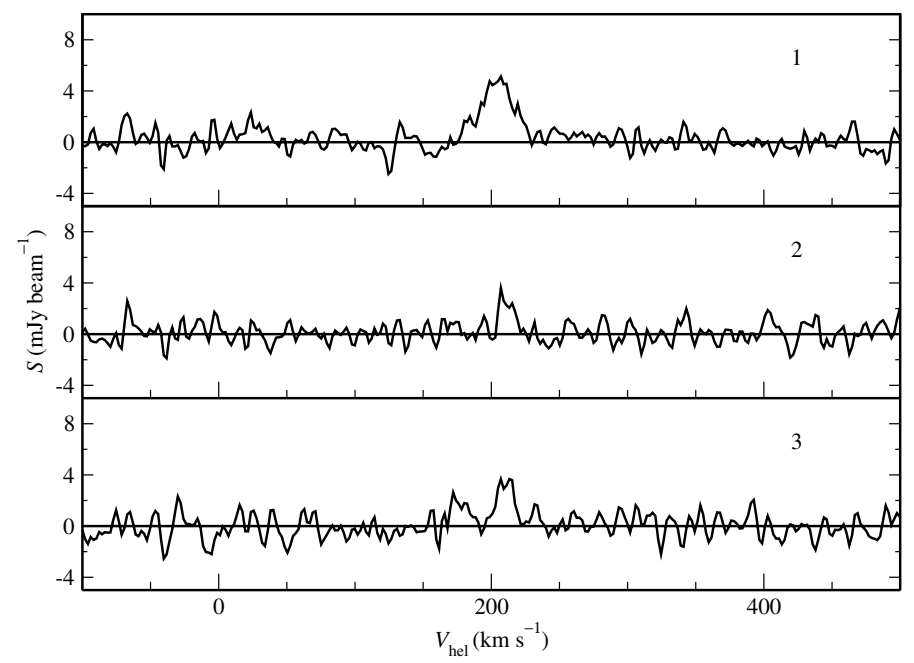

Fig. 4. Spectra taken at the positions indicated in Fig. 3.

\section{Nature of GBT 1355}

In the following, we discuss a number of possibilities for the nature of GBT 1355. One obvious scenario is that it is an object in the vicinity of M101. However, because only one dark H I cloud is detected near M101, while the sensitivity of the data is good enough to have detected much fainter clouds as well, GBT 1355 may have no physical relation to M101 and the proximity to M101 on the sky could be a chance superposition. Therefore, we also discuss the possibilities that GBT 1355 is a foreground object in the Galactic halo or in the Local Group.

\subsection{Object near M101}

First we discuss the possibility that GBT 1355 is an object associated with M101. If this is the case ${ }^{1}$, its size would be $10 \times 6 \mathrm{kpc}$ and the HI mass $1.1 \times 10^{7} M_{\odot}$. The projected distance to the centre M101 would be $150 \mathrm{kpc}$.

One possibility is that GBT 1355 is a remnant of a tidal interaction with one of the members of the M101 group. Such remnants are seen near many large galaxies (see e.g., Sancisi et al. 2008). Mihos et al. (2012) discussed this possibility and concluded that there is no observational evidence that GBT 1355 is associated with an ongoing or recent tidal interaction. While GBT 1355 is detected with high $\mathrm{S} / \mathrm{N}$ in their data, they did not detect any other $\mathrm{H}$ I that could hint at a tidal nature of GBT 1355. In addition, in the deep optical images of Mihos et al. (2013) no indications for such an interaction were found either.

We can add to this that the shape and kinematics do not quite match that what is generally expected for tidal filaments. Because of the nature of tidal forces, tidal filaments are usually linear structures, different from GBT 1355. Moreover, velocity gradients in tidal filaments tend to be aligned with such filaments, while the velocity gradient in GBT 1355 is perpendicular.

If GBT 1355 is not a tidal filament, it could be a "proper" galaxy near M101. However, it would be then have to be of exceptionally low optical surface brightness. For example, in the compilation of dwarf galaxies in and around the Local Group of McConnachie (2012), all dwarfs detected in H I have central surface brightnesses brighter than $26 \mathrm{mag} \operatorname{arcsec}^{-2}$ in $V$, with most brighter than $\mu_{\circ, V}=24 \mathrm{mag} \operatorname{arcsec}^{-2}$. Any optical emission from

\footnotetext{
1 We assume the same distance to M101 as in Mihos et al. (2012): $6.9 \mathrm{Mpc}$. For this distance, 1 arcmin corresponds to $2.0 \mathrm{kpc}$.
}

GBT 1355 must be much fainter: the upper limit to the $V$ surface brightness is 29 mag $\operatorname{arcsec}^{-2}$ (Mihos et al. 2013).

Assuming GBT 1355 is a gravitationally bound object, we can estimate its total dynamical mass. One uncertainty is that we do not know the shape of the total matter distribution, and therefore do not know exactly how to use the kinematical information. A frequently used compromise is (Hoffman et al. 1996)

$M_{\text {dyn }}=2.325 \times 10^{5}\left(\frac{V_{\mathrm{rot}}^{2}+3 \sigma^{2}}{\mathrm{~km}^{2} \mathrm{~s}^{2}}\right)\left(\frac{r}{\mathrm{kpc}}\right) M_{\odot}$.

Another complication is that one does not know whether to interpret the velocity gradient as rotation, and even if one could do so, the inclination would not be well constrained. We therefore derived a lower limit to the dynamical mass by assuming that there is no systematic rotation. In this way, we found $M_{\text {dyn }}>3.5 \times 10^{8} M_{\odot}$, using $\sigma=10 \mathrm{~km} \mathrm{~s}^{-1}$ and $r=5 \mathrm{kpc}$. Except for the case where GBT 1355 is seen fairly face on, the effect of possible rotation is modest. For example, assuming that the velocity gradient indicates rotation and, in addition, the inclination is $30^{\circ}$, we find $M_{\text {dyn }}=6.1 \times 10^{8} M_{\odot}$.

Interestingly, if GBT 1355 is a dark object near M101, its properties would be very similar to those of the compact highvelocity clouds (CHVCs) in the model proposed by Braun \& Burton (1999). In this model, the observed CHVCs are a population of unevolved (dark) dwarf galaxies scattered throughout the Local Group, at distances up to $1 \mathrm{Mpc}$. This model has met with a number of objections, however. One is that such dark objects would be too large to be consistent with theoretical models of small, starless dark matter halos containing observable H I (Sternberg et al. 2002; Maloney \& Putman 2003). Another objection is that surveys of nearby galaxy groups have not detected similar populations of similarly sized H I clouds (e.g. Zwaan \& Briggs 2000; Pisano et al. 2007; Chynoweth et al. 2011). The same objections would apply here. According to the CHVC model of Braun \& Burton (1999), one would expect a substantial population of failed dwarf galaxies near M101, but the data of Mihos et al. (2012) exclude this.

\subsection{Galactic object}

The above discussion shows that there could be some problems with the scenario of GBT 1355 as an object in the direct vicinity of M101. We therefore discuss two other possibilities, the first one being that GBT 1355 is an H I cloud associated with our own Galaxy. Given its high Galactic latitude $\left(60^{\circ}\right)$ and its velocity, GBT 1355 would then be a CHVC somewhere in the Galactic halo. If so, it would be quite a small cloud: for a distance of $10 \mathrm{kpc}$, the physical size would be $14.5 \times 8.7 \mathrm{pc}$ and its H I mass only $24.7 M_{\odot}$.

However, if GBT 1355 is Galactic, it would be a somewhat unusual CHVC. As Mihos et al. (2012) discussed, within a radius of several tens of degrees from GBT 1355 there are indeed a number of known Galactic HVCs, but they are at quite different velocities. Almost all these HVCs have negative velocities. The highest velocity of a Galactic HVC in this region of sky is $+50 \mathrm{~km} \mathrm{~s}^{-1}$ (see Wakker \& van Woerden 1991) therefore the velocity differences between GBT 1355 and the nearby HVCs are at least $150 \mathrm{~km} \mathrm{~s}^{-1}$, ruling out any association. However, the observations used by Wakker \& van Woerden (1991) are not deep enough to have detected small clouds like GBT 1355, so the possibility remains that there is a population of smaller CHVCs in this region of the sky. Mihos et al. (2012) noted, however, that they found no trace of any other Galactic H I that could be associated with GBT 1355 in their deep GBT observations. 
Some additional information comes from the kinematics. Imaging studies of CHVCs have shown (e.g. de Heij, Braun \& Burton 2002; Brüns \& Westmeier 2004) that they typically consist of cores with line widths of at most a few $\mathrm{km} \mathrm{s}^{-1}$ that are embedded in more diffuse emission with velocity widths similar to those seen in GBT 1355. GBT 1355 does appear to consist of gas condensations surrounded by more diffuse gas. However, a distinct difference seems to be that the profiles of these condensations are much broader than those of the cores of CHVCs. On the other hand, we cannot entirely exclude that the sensitivity of our data is not good enough to have detected such narrow components. We have planned more sensitive observations to investigate this in more detail.

We note that GBT 1355 is located on the great circle spanned by the Magellanic stream (Nidever et al. 2010). As seen from the Magellanic Clouds, it is about 90 degrees beyond the tip of the leading arm of the Magellanic H I system. Although the extent of the leading arm has not been completely determined, this makes an association with the leading arm unlikely.

\subsection{Local group minihalo}

The last hypothesis we discuss is that GBT 1355 is a foreground object located in the Local Group. This is, in fact, an interesting possibility. Giovanelli et al. (2010) and Adams et al. (2013) reported the discovery of a number of ultra-compact HVCs with properties that, although other interpretations cannot be entirely excluded, are consistent with them being isolated, dark minihalos in the outskirts of the Local Group. If they are assumed to be at a distance of $1 \mathrm{Mpc}$, these clouds have H I masses in the range of $10^{5} M_{\odot}$ to $10^{6} M_{\odot}$, sizes up to a few $\mathrm{kpc}$, and dynamical masses of $10^{7}-10^{8} M_{\odot}$. Their heliocentric velocities range up to $+320 \mathrm{~km} \mathrm{~s}^{-1}$ and they have linewidths of about 20$30 \mathrm{~km} \mathrm{~s}^{-1}$. They are basically smaller versions, by roughly a factor 10, of the clouds of the CHVC model of Braun \& Burton (1999). However, the clouds as proposed by Giovanelli et al. (2010) and Adams et al. (2013) are consistent with the theoretical models of, e.g., Sternberg et al. (2002). They would also be small enough to have escaped detection in existing searches for dark $\mathrm{H}$ I clouds in nearby groups of galaxies.

Assuming GBT 1355 is at a distance of $1 \mathrm{Mpc}$, its properties would be similar to those deduced for these clouds. Its H I mass would be $2.4 \times 10^{5} M_{\odot}$, its size $1.4 \times 0.9 \mathrm{kpc}$, and the dynamical mass $M_{\text {dyn }}>5 \times 10^{7} M_{\odot}$. The number density of clouds reported by Adams et al. (2013) is such that one would expect about one such cloud in the survey area of Mihos et al. (2012).

One useful comparison is with very small, almost dark, H Idominated galaxies such as Leo T (Ryan-Weber et al. 2008) and Leo P (Giovanelli et al. 2013). The size, gas content, and dynamical mass of these galaxies are very similar to that of GBT 1355, if it is assumed to be at $1 \mathrm{Mpc}$. The two Leo galaxies do have an observable number of stars, but they are completely gas dominated. Their stellar components are very faint and $>70 \%$ of the visible baryons are in HI. Therefore, the stellar components are not very relevant for their overall structure. If GBT 1355 were a dark minihalo, one would expect that it would be very similar kinematically. This is not the case. Both Leo $\mathrm{T}$ and Leo P do not have a clumpy $\mathrm{H}$ I distribution and show not much structure in the velocity dispersions. This could argue against the minihalo hypothesis, unless GBT 1355 happens to be a bound pair of minihalos. H I imaging of the Giovanelli and Adams clouds will be useful to investigate this question in greater detail. The radial velocity and sky location are consistent with GBT 1355 being a member of the loose group containing Leo P.

\section{Summary}

We have imaged the H I in GBT $1355+5439$, a starless H I cloud near M101 recently discovered by Mihos et al. (2012). We find it to be a cloud of $5 \times 3 \mathrm{arcmin}$. The distribution and kinematics indicate that it consists of a small number of gas concentrations that have small relative motions with respect to each other. Because the distance to GBT 1355 is not known, we discussed a number of possibilities for the nature of this cloud. However, none of these provided a perfect explanation. If the object is assumed to be physically associated with M101, we find that the size, structure and kinematics is not what one would expect for either a tidal filament near M101, or for a dark, starless galaxy in the M101 group. Although the data neither exactly fit the properties of known CHVCs in the Galactic halo, we cannot entirely exclude this option. Deeper observations are required for a more detaild study. If the object is part of the Local Group, its size and mass would be very similar to that of the clouds from the Local Group population of $\mathrm{H}$ I objects recently proposed by Giovanelli et al. (2010) and Adams et al. (2013). In that scenario, it would also have similar size and mass as that of some of the smallest known gas-dominated dwarf galaxies in or near the Local Group and it would only differ by the absence of a very small population of stars. However, the internal kinematic of GBT 1355 seems to be different from such objects.

Acknowledgements. We thank Chris Mihos for providing the deep optical images of GBT $1355+5439$ in digital form. We also thank the anonymous referee for valuable input. The Westerbork Synthesis Radio Telescope is operated by the ASTRON (Netherlands Institute for Radio Astronomy) with support from the Netherlands Foundation for Scientific Research (NWO). W.J.Gd.B. was supported by the European Commission Grant FP7-PEOPLE-2012-CIG\#333939

\section{References}

Adams, E. A. K., Giovanelli, R., \& Haynes, M. P. 2013, ApJ, 768, 77

Bovill, M. S., \& Ricotti, M. 2011, ApJ, 741, 18

Braun, R., \& Burton, W. B. 1999, A\&A, 341, 437

Brüns, C., \& Westmeier, T. 2004, A\&A, 426, L9

de Heij, V., Braun, R., \& Burton, W. B. 2002, A\&A, 391, 67

Chynoweth, K. M., Holley-Bockelmann, K., Polisensky, E., \& Langston, G. I. 2011, AJ, 142, 137

Giovanelli, R., Haynes, M. P., Kent, B. R., \& Adams, E. A. K. 2010, ApJ, 708, L22

Giovanelli, R., Haynes, M. P., Adams, E. A. K., et al. 2013, AJ, 146, 15

Hoffman, G. L., Salpeter, E. E., Farhat, B., et al. 1996, ApJS, 105, 269

Klypin, A., Kravtsov, A. V., Valenzuela, O., \& Prada, F. 1999, ApJ, 522, 82

Maloney, P. R., \& Putman, M. E. 2003, ApJ, 589, 270

McConnachie, A. W. 2012, AJ, 144, 4

Meyer, M. J., Zwaan, M. A., Webster, R. L., et al. 2004, MNRAS, 350, 1195

Mihos, J. C., Keating, K. M., Holley-Bockelmann, K., Pisano, D. J., \& Kassim, N. E. 2012, ApJ, 761, 186

Mihos, J. C., Harding, P., Spengler, C. E., Rudick, C. S., \& Feldmeier, J. J. 2013 , ApJ, 762, 82

Moore, B., Ghigna, S., Governato, F., et al. 1999, ApJ, 524, L19

Nidever, D. L., Majewski, S. R., Butler Burton, W., \& Nigra, L. 2010, ApJ, 723, 1618

Pisano, D. J., Barnes, D. G., Gibson, B. K., et al. 2007, ApJ, 662, 959

Ricotti, M. 2009, MNRAS, 392, L45

Ryan-Weber, E. V., Begum, A., Oosterloo, T., et al. 2008, MNRAS, 384, 535

Sancisi, R., Fraternali, F., Oosterloo, T., \& van der Hulst, T. 2008, A\&ARv., 15, 189

Sault, R. J., Teuben, P. J., \& Wright, M. C. H. 1995, in Astronomical Data Analysis Software and Systems IV, eds. R. Shaw, H. E. Payne, \& J. J. E. Hayes, ASP Conf. Ser., 77, 433

Schaye, J. 2004, ApJ, 609, 667

Sternberg, A., McKee, C. F., \& Wolfire, M. G. 2002, ApJS, 143, 419

Wakker, B. P., \& van Woerden, H. 1991, A\&A, 250, 509

Zwaan, M. A., \& Briggs, F. H. 2000, ApJ, 530, L61 